\title{
Kinetics of reactive oxygen species produced by rainbow trout (Oncorhynchus mykiss) leukocytes and the effect of the antioxidant astaxanthin
}

\author{
Leonardo Gomez ${ }^{1}$, Raul Cortes ${ }^{2}$, Ariel Valenzuela ${ }^{3}$, Carlos Smith ${ }^{1}$ \\ ${ }^{1}$ University of Concepcion, Faculty of Biological Sciences, Department of Microbiology Concepcion, Chile \\ ${ }^{2}$ Consejo Superior de Investigaciones Cientificas (CSIC), Instituto de Acuicultura de Torre de la Sal (IATS), \\ Department of Fish Physiology and Biotechnology, Castellon, Spain \\ ${ }^{3}$ University of Concepcion, Faculty of Natural Sciences and Oceanography, Department of Oceanography, \\ Concepcion, Chile
}

Received December 17, 2012

Accepted August 28, 2013

\begin{abstract}
Reactive oxygen species contribute to kill of microorganisms. Their activity is usually measured by their capacity to reduce nitroblue tetrazolium into formazan. The incubation time to allow nitroblue tetrazolium reduction by reactive oxygen species usually ranges from 30 to $60 \mathrm{~min}$. The aim of our study was to determine the kinetics of formazan formation, to determine the shortest incubation time possible, and to find if astaxanthin negatively affects the availability of reactive oxygen species (and defense mechanisms of fish). The blood/nitroblue tetrazolium method is based on nitroblue tetrazolium reduction into formazan by reactive oxygen species present in blood. Formazan can be spectrophotometrically measured, allowing quantification of reactive oxygen species. Reactive oxygen species were measured in blood samples from 30 trout intramuscularly injected with astaxanthin $(0.3 \mathrm{mg} / 100 \mathrm{~g}$ of fish) (experimental group) and 30 non-injected trout (controls). Results indicated that in trout non-treated with astaxanthin, the plateau of formazan production was reached after 20 min of incubation. Trout intramuscularly injected with astaxanthin showed the following: on Day 1 after astaxanthin injection, the kinetics were slower but finally reached a plateau similar to astaxanthin-free trouts, and by Day 11 the plateau was significantly higher after $60 \mathrm{~min}$ incubation. In conclusion, the kinetics curves here reported allow reducing incubation time of the method to only $20 \mathrm{~min}$ in antioxidant-free trout and, on the other hand, our results also revealed that astaxanthin can be used to improve flesh colour in salmonids without affecting reactive oxygen species availability and therefore the defense mechanisms of trout.
\end{abstract}

Fish, innate immunity, respiratory burst, NBT

Fish innate immune response, their first defensive line against pathogens, is considered essential to protect them against infectious diseases (Magnadóttir 2006), particularly in the acute form. Cellular components of the innate response include neutrophils and macrophages, two types of leukocytes using the "respiratory burst" as one of their mechanisms to destroy microorganisms. The respiratory burst produces several highly toxic molecules, including reactive oxygen species (ROS), usually called oxidative radicals, such as superoxide anion $\left(\mathrm{O}_{2}^{-}\right)$and hydrogen peroxide $\left(\mathrm{H}_{2} \mathrm{O}_{2}\right)($ Secombes 1996 ; Lundén et al. 2002). A decrease in ROS production can negatively affect the antibacterial activity of the host (Secombes 1996), demonstrating their importance to eliminate pathogenic microorganisms. Several substances can increase or decrease ROS production (Sakai 1999; Puangkaew et al. 2004; Kumari and Sahoo 2006; Ispir et al. 2008), thus positively or negatively affecting the ability of the animal to protect itself against an infection. As mentioned above, a decrease in ROS may turn an organism more susceptible to infection. Antioxidants may react with ROS, depriving animals of these compounds used by cells, such as leukocytes, to destroy microorganisms. Therefore, antioxidants might negatively affect the host's defense.

Carotenoid astaxanthin is added to fish diet to provide attractive colour to salmon flesh, but it is also a powerful antioxidant, $100 \times$ more efficient than vitamin E (Miki 1991), able

Address for correspondence:

Carlos T. Smith

University of Concepcion, Faculty of Biological Sciences

Department of Microbiology

P. O. Box 160-C, Concepcion, Chile

Phone: 56 (41) 2204287

Fax: 56 (41) 2245975

E-mail: csmith@udec.cl

http://actavet.vfu.cz/ 
to inactivate oxidizing radicals, thus avoiding tissue damage. Therefore, astaxanthin added to diet could compete with ROS and thus consume ROS that otherwise might be used to destroy microorganisms, negatively affecting innate immunity of fish.

Reactive oxygen species have usually been assessed using the blood/nitroblue tetrazolium (NBT) method, based on the reduction of NBT by ROS into the colored compound formazan, the amount of which is proportional to available ROS and can be spectrophotometrically measured. Formazan produced by NBT reduction mediated by ROS has traditionally been measured after 30 (Anderson and Siwicki 1993; Kumari and Sahoo 2006; Ispir et al. 2008), 45 (Valenzuela et al. 2005) or 60 min incubation (Secombes 1990; Logambal et al. 2000; Puangkaew et al. 2004), but we have not been able to find justification for the incubation time used. Therefore, study of formazan formation kinetics was necessary to determine the optimal incubation time.

This study was therefore conducted with regard to two aims. Firstly, to know the kinetics of NBT reduction into formazan in a fish leukocyte model in order to determine the shortest incubation time for this technique. Secondly, to determine if the powerful antioxidant astaxanthin consumes ROS that otherwise would be available for host defense purposes.

\section{Materials and Methods}

Fish and experimental design

Sixty apparently healthy juvenile rainbow trout (170-289 g, mean $242 \mathrm{~g} \pm 35.8 \mathrm{SD})$ of both sexes were kept at the "Salmones Pangue" pisciculture (Bio-Bio Region, Chile). Fish were placed in two 1,000 1 tanks (30 animals in each one) with biomass of approximately $6 \mathrm{~g} / \mathrm{l}$, water exchange of $15 \mathrm{l} / \mathrm{min}$ and under natural spring photoperiod. Fish were maintained in their respective tanks for one month before starting the experiment. During this time and during the experiment, they were fed a commercial feed (EWOS transfer 250, astaxanthin-free) at a rate of 3\% of the biomass.

Fish $(n=30)$ from one tank (experimental group) were injected intramuscularly with the carotenoid astaxanthin (3,3'dihydroxy- $\beta, \beta$ carotene-4,4'dione) in vegetal oil at a dose of $0.3 \mathrm{mg} / 100 \mathrm{~g}$ of fish on Day 0 of experiment. Astaxanthin was kindly provided by Astax S.A. (Iquique, Chile). Vegetal oil had no effect on the indicators here evaluated (data not shown). Fish $(\mathrm{n}=30)$ from the other tank (control group) were injected intramuscularly with saline solution on Day 0 of experiment. Fish from both tanks were used for sampling on days 1, 7 and 11 of the experiment (nine fish sampled at each sampling day).

Fish were anaesthetized with $15 \mathrm{ml}$ of $20 \% \mathrm{w} / \mathrm{v}$ benzocaine (Veterquimica, Chile) in 1001 of water. Blood was drawn by caudal puncture using heparinized syringes, transferred to Eppendorf tubes and kept at $4{ }^{\circ} \mathrm{C}$ until arrival at the Immunology Laboratory of the Department of Microbiology, University of Concepcion (Concepcion, Chile) within $1 \mathrm{~h}$ of sampling. At the end of the experiment, necropsies were performed randomly to one third of the animals of each tank (10 animals per tank) to evaluate their health condition and all of them were apparently healthy.

Reactive oxygen species quantification and kinetics

From the blood samples, ROS were quantified by means of the blood/NBT method slightly modified from Anderson and Siwicki (1993). Briefly, $0.1 \mathrm{ml}$ blood was incubated with $0.1 \%$ NBT (Sigma Chemical Co., St. Louis, MO, USA) in saline. In order to obtain the kinetics, we used measurements at 5, 10, 15, 20, 30, 40, 45 and $60 \mathrm{~min}$ instead of only 60,45 or $30 \mathrm{~min}$ of incubation time. After the completion of incubation, $1 \mathrm{ml}$ of N,N-dimethylformamide (Merck, Darmstadt, Germany) was added to the blood/NBT mixture and centrifuged for $10 \mathrm{~min}$ at $100 \times \mathrm{g}$. Reactive oxygen species were quantified by spectophotometric measurement of formazan in the supernatant at $620 \mathrm{~nm}$. The same technique was used for both astaxanthin-free and astaxanthin-injected animals. In all procedures, care was taken to avoid astaxanthin oxidation by light.

Data analysis

Formazan formation kinetics from astaxanthin non-injected and injected fish were analyzed by means of a twoway ANOVA with a significance level of 0.05 .

\section{Results}

As shown in Fig. 1, ROS of astaxanthin-free fish, measured by means of formazan accumulation, reach the plateau after $20 \mathrm{~min}$ of blood/NBT incubation. After that, no significant increase of absorbance (formazan accumulation) occurred. 


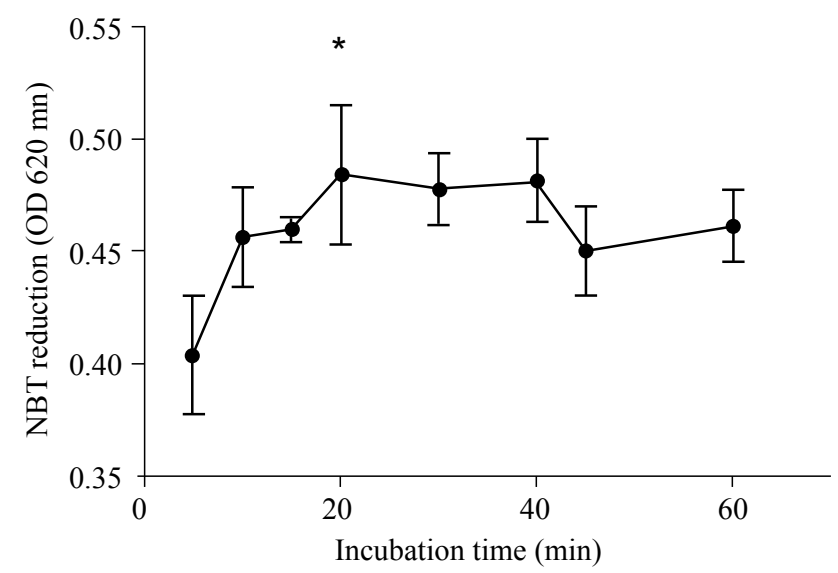

Fig. 1. Kinetics of nitroblue tetrazolium reduction by peripheral blood reactive oxygen species of astaxanthin-free rainbow trout.

NBT - nitroblue tetrazolium, OD - optical density. Data are expressed as mean \pm standard deviation $(\mathrm{n}=9$ in each incubation time). ${ }^{*}$ Time when NBT reduction (formazan formation) reached the plateau.

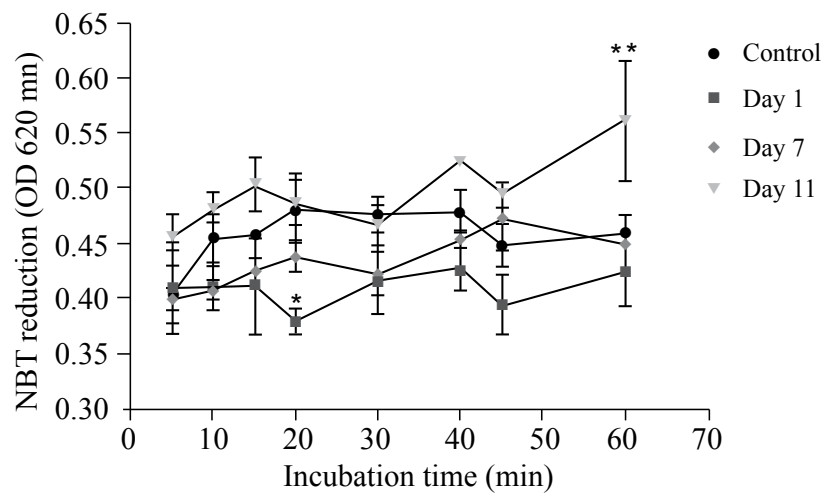

Fig. 2. Effect of astaxanthin on nitroblue tetrazolium reduction at days 1, 7 and 11 after injection of astaxanthin to fish and compared to astaxanthin-free fish.

NBT - nitroblue tetrazolium, OD - optical density. Data are expressed as mean \pm standard deviation $(\mathrm{n}=9$ in each incubation time). * Shows significant decrease $(P<0.05), * *$ shows significant increase $(P<0.05)$.

Formazan formation was assessed at the same incubation periods $(5,10,15,20,30,40$, 45 and $60 \mathrm{~min}$ ) at days 1,7 and 11 in astaxanthin-injected animals. When compared to astaxanthin-free animals (Fig. 2), formazan accumulation showed a significant decrease at 20 min on Day 1. A significant increase was also observed in astaxanthin-injected animals at Day 11 after 60 min of incubation. 


\section{Discussion}

Blood of astaxanthin-free trout showed a rapid reduction of NBT into formazan, reaching the plateau at $20 \mathrm{~min}$ of incubation. After that $(30,40,45$ and $60 \mathrm{~min})$ there was no significant absorbance increase. Therefore, at least in this animal model, $20 \mathrm{~min}$ is the minimum incubation time for the mixture blood/NBT. It is shorter than that reported in the literature to evaluate the "respiratory burst" which is usually 45 min (Valenzuela et al. 2005) or 60 min (Secombes 1990; Logambal et al. 2000; Puangkaew et al. 2004). This 20 min incubation time is even shorter than that of $30 \mathrm{~min}$ used by other authors (Anderson and Siwicki 1993; Kumari and Sahoo 2006; Ispir et al. 2008). Therefore, the contribution of this work is to report the kinetics of this reaction, providing a solid base to define the incubation time for future ROS measurements in rainbow trout. It will be interesting to determine if this incubation time is appropriate in other animal models.

In astaxanthin-injected fish, the kinetics of NBT reduction into formazan showed significant changes on two occasions. One of them was a decrease in ROS activity at 20 min of incubation on Day 1 although it recovered to control levels at the end of the 60 min incubation time, making this decrease transient. Kinetics was slower but it finally reached the same oxidative power as the control. The slower ROS activity on Day 1 might be caused by the powerful antioxidant activity of this carotenoid (Miki 1991) injected on the previous day. This effect was not evident on Days 7 and 11 after astaxanthin injection. Therefore, if astaxanthin is capable of temporarily decreasing microbicidal activity in fish when challenged by pathogenic microorganisms, it should not affect its immune defenses in the long run.

The second difference was a general increase in ROS availability on Day 11 which was significant by the end of the 60 min period. A possible explanation is that the transient decrease in ROS caused by astaxanthin on Day 1 might cause a physiological response producing a ROS increase ("rebound") on the following days. Therefore, at least for a few days after the first week post-injection, astaxanthin might favour antimicrobial activity. It must be pointed out that astaxanthin has been reported to have a positive effect on other indicators of the defense mechanisms (Jyonouchi et al. 1995; Chew et al. 1999).

In conclusion, blood/NBT incubation time can be reduced to $20 \mathrm{~min}$ in this fish model, and perhaps in other models, when it is free of astaxanthin. A longer blood/NBT incubation time is necessary in trout receiving astaxanthin or possibly other antioxidants. Secondly, the astaxanthin effect on the availability of ROS seems to be transient and not detrimental to the defense mechanisms.

\section{Acknowledgements}

This work was supported in part by Grant 97.036.05-1, Direccion de Investigacion, Universidad de Concepcion. We are grateful to Mr. Ricardo Quiroz (owner of Salmones Pangue) and to Mr. Mario Pizarro (Astax-Chile), who provided us the astaxanthin.

\section{References}

Anderson D, Siwicki A 1993: Measuring the effects of contaminants on fish by haematological and serological methods. In: Stolen J, Anderson D, Zelikoff S, Twerdok L, Kaattari S (eds): Modulators of fish immune responses. SOS Publications, Fair Haven NJ, pp. 95-118

Chew B, Wong M, Park J, Wong T 1999: Dietary beta-carotene and astaxanthin but not canthaxanthin stimulate splenocyte function in mice. Anticancer Res 19: 5223-5227

Ispir U, Bayram H, Ozcan M, Dorucu M, Saglam N 2008: Immune response of rainbow trout (Oncorhynchus mykiss) to selected antigens of Yersinia ruckeri. Acta Vet Brno 78: 145-150

Jyonouchi H, Sun S, Gross M 1995: Astaxanthin, a carotenoid without vitamin A activity, augments antibody responses in cultures including T-helper cell clones and suboptimal doses of antigen. J Nutr 125: 2483-2492

Kumari J, Sahoo P 2006: Dietary levamisole modulates the immune response and disease resistance of Asian catfish Clarias batrachus (Linnaeus). Aquac Res 37: 500-509 
Logambal S, Venkatalakshmi S, Michael R 2000: Immunostimulatory effect of leaf extract of Ocimum sanctum Linn. in Oreochromis mossambicus (Peters). Hydrobiologia 430: 113-120

Lundén T, Lilius E, Bylund G 2002: Respiratory burst activity of rainbow trout (Oncorhynchus mykiss) phagocytes is modulates by antimicrobial drugs. Aquaculture 207: 203-213

Magnadóttir B 2006: Innate immunity of fish (overview). Fish Shellfish Immunol 20: 137-151

Miki W 1991: Biological functions and activities of animal carotenoids. Pure Appl Chem 63: 141-146

Puangkaew J, Kiron V, Somamoto T, Okamoto N, Satoh S, Takeuchi T, Watanabe T 2004: Nonspecific immune response of rainbow trout (Oncorhynchus mykiss Walbaum) in relation to different status of vitamin $\mathrm{E}$ and highly unsaturated fatty acids. Fish Shellfish Immunol 16: 25-39

Sakai M 1999: Current research status of fish immunostimulants. Aquaculture 172: 63-99

Secombes C 1990. Isolation of salmonid macrophages and analysis of their killing activity. In: Stolen J, Fletcher T, Anderson D, Robertson B, Van Muiswinkel W (eds): Techniques in fish immunology. SOS Publications, Fair Haven, NJ, pp. 37-154

Secombes C 1996. The nonspecific immune system: cellular defences. In: Iwama G and Nakanishi T (eds.): The fish immune system: organism, pathogen and environment. Academic Press Ltd., London, pp. 63-103

Valenzuela A, Silva V, Tarifeño E, Klempau A 2005: Effect of acute hypoxia in trout (Oncorhynchus mykiss) on immature erythrocyte release and production of oxidative radicals. Fish Physiol Biochem 31: 65-72 